\title{
Skén\&graphie
}

SKIINaGRAPIII Coulisses des arts du spectacle et des scènes

émergentes

$5 \mid 2018$

Juste la fin du monde, de Lagarce à Dolan

\section{Ce que signifie « juste » dans Juste la fin du monde}

\section{Maryse Adam-Maillet}

\section{(2) OpenEdition \\ Journals}

Édition électronique

URL : http://journals.openedition.org/skenegraphie/1358

DOI : 10.4000/skenegraphie.1358

ISSN : 2553-1875

\section{Éditeur}

Presses universitaires de Franche-Comté

\section{Édition imprimée}

Date de publication : 1 janvier 2018

Pagination : 23-32

ISBN : 978-2-84867-5609-8

ISSN : $1150-594 X$

\section{Référence électronique}

Maryse Adam-Maillet, « Ce que signifie « juste » dans Juste la fin du monde », Skén\&graphie [En ligne], 5 | 2018, mis en ligne le 01 janvier 2019, consulté le 10 décembre 2020. URL : http:// journals.openedition.org/skenegraphie/1358; DOI : https://doi.org/10.4000/skenegraphie.1358

Ce document a été généré automatiquement le 10 décembre 2020.

Presses universitaires de Franche-Comté 


\title{
Ce que signifie « juste » dans Juste la fin du monde
}

\author{
Maryse Adam-Maillet
}

1 Observer Juste la fin du monde à partir du prisme de la réécriture, c'est rassembler un ample matériau génétique ou intertextuel, en amont ou en aval de la version actuelle de la pièce. Avant même de concerner la question de la transposition du théâtre au cinéma dans la circonstance de la sortie du film de Dolan ${ }^{1}$, la réécriture informe la pièce ellemême, dans sa relation avec le reste de la création lagarcienne : elle y occupe une place particulière en termes de lutte pour la création. Dans cette entreprise de réécriture interne, organique de l'œuvre, le titre Juste la fin du monde, constitue non seulement la matrice mais encore la condition de possibilité de la production de la pièce dans son état final. Je me dois aussi de préciser ce que l'hypothèse d'une telle fonction heuristique du titre dans le processus de création doit à Sylvie Thorel, chercheure en littérature française et comparée ${ }^{2}$.

2 Juste la fin du monde, texte académique, classique, forme un chef d'œuvre, économique et virtuose à la fois, dans l'acception de l'artisanat et du métier, et vraisemblablement aussi au sens axiologique du terme. Aucun hasard : la pièce entre la première dans le répertoire de la Comédie Française ${ }^{3}$ et fait l'objet d'une adaptation cinématographique qui connait le succès que l'on sait. Or, la faveur de la réception actuelle semble inversement proportionnelle à la difficulté créatrice marquant les années d'élaboration.

3 La genèse et la première réception de Juste la fin du monde - entre 1988 et 1993 stricto sensu (mais en réalité, il y a nécessité de prendre en compte la période de fin 1986 à 1990) correspond pour l'auteur à un moment de très grande crise personnelle, de panne dans la création littéraire. La pièce n'est pas reçue de son vivant; il écrit peu et difficilement avant (Les Prétendants unique pièce aboutie, Les Adieux avec une peine et une contention infinie), et il écrit peu et difficilement après : deux ans au total sans écriture personnelle.

Une étrangeté frappe dans la période critique du laboratoire de Juste la fin du monde: Les Adieux ont été le premier titre de la pièce (première idée de la pièce en 1988), ainsi que le second titre du récit que l'on connaît sous ce nom aujourd'hui et qu'on appelle parfois « le roman » de Lagarce (première idée du récit en 1986). Lagarce lui-même parle de version 
des Adieux pour le théâtre ${ }^{4}$, de version théâtrale du récit. L'anomalie réside dans le fait que, lus successivement, les deux textes semblent n'entretenir aucun rapport. Le second n'a rien d'un sous-produit du premier, d'un fragment détaché ou d'une suite, d'un complément: pas de lien visible entre ces échantillons de genres éloignés, seulement, peut-être, la présence forte du personnage d'Antoine. La relation entre le récit et la pièce, aux écritures contiguës, est cependant explicitement posée par Lagarce à plusieurs reprises, comme remplissant la même fonction littéraire d'Adieux, investie des mêmes enjeux, «Tenter de faire mes adieux avec Quelques éclaircies. Juste après ${ }^{5}$. » Les deux textes interrogent l'impossibilité à écrire, à faire œuvre, à être lu, à laisser une trace pour la postérité. Ils constituent une sorte de pari ordalique, "ça passe ou ça casse ", qui a fonctionné pour Juste la fin du monde mais pas pour Les Adieux, resté inédit. Trivialement, il est exact de dire que Les Adieux ça casse, mais que Juste la fin du monde, ça passe. Les deux productions se donnent pour des œuvres ultimes, chargées d'apporter la preuve définitive et in extremis du statut d'écrivain de littérature à la lumière de la mort annoncée à court terme. Paradoxalement, nihil novi dans l'œuvre, mais une tentative de cristallisation de l'existant. Le rapport entre le récit inédit Les Adieux et la pièce Juste la fin $d u$ monde procède aussi du rapport général entre tous les textes comme autant de parties du corps d'une œuvre dont la mort permettra l'aboutissement et la révélation posthume de la vie organique, à la manière de la dialectique explorée dans Les Mots par Sartre.

J'ai pensé aussi que j'étais trop pressé et que l'œuvre littéraire prendrait la vie entière et que je ne saurais jamais rien en fait de son intérêt ${ }^{6}$.

L'œuvre tout entière (pièces / journal / récit) lie crise, angoisse face à la mort, et récriture, ambition esthétique. La présence de la maladie, son envahissement commencent bien avant la maladie réelle, dans la connivence du rapport aux garçons et de la mort, la connivence du rapport dangereux aux guerriers et de la mort, dans le pacte d'Eros et de Thanatos. C'est vrai depuis toujours, bien avant la déclaration officielle du diagnostic de 1988 et l'annonce de la mort certaine et proche par la maladie. Deux grandes obsessions héroïques organisent cette économie psychique : la fin et le retour. Que l'on examine les titres, y compris Histoire d'amour (Derniers chapitres), qui montrent, techniquement, le mouvement de réécriture perpétuelle dans l'œuvre personnelle, par exemple :

- 12 juillet 86 : «Incapacité d'écrire »7. Projet de la deuxième version d'Histoire d'amour (sous le titre Derniers chapitres au constat amoureux) ;

- de ce projet sort d'abord Dernier remords avant l'oubli qui comporte les personnages d'Antoine et d'Hélène : " c'est méchant, un peu violent, dur " ${ }^{8}$;

- suit le récit Les Adieux où l'on retrouve les personnages d'Antoine et d'Hélène, tout aussi méchant, dur, violent ;

- puis Juste la fin du monde, avec les personnages de Louis et d'Antoine ;

- variation: J'étais dans ma maison et j'attendais que la pluie vienne écrit en 1994 ; le héros revenant meurt (comme une variante de l'épilogue de Juste la fin du monde ?);

- enfin, Le Pays lointain, englobement et amplification de Juste la fin du monde.

6 Si l'on en revient maintenant aux données factuelles de la réécriture entre Les Adieux et Juste la fin du monde, on peut se fonder sur l'évidence du jeu des titres pour le récit: Le Combat avec l'Enfant ${ }^{9}$ / Mes deux dernières années / Les Adieux. Pour la pièce : Les Adieux / Et quelques éclaircies / Quelques éclaircies / Juste à la fin du monde / Juste la fin du monde. Il est probable que la lecture de Mes parents joue un rôle important dans ce double laboratoire récit-pseudo-roman / théâtre. Hervé Guibert était présent au four de De Saxe, roman, en 
1985 (l'épisode correspond au début du récit qui porte le titre Les Adieux). La référence à ce livre-là, Mes parents, quand Lagarce " autopsie » ses propres parents dans Les Adieux, s'impose. La figure de Guibert, écrivain génial dont on sait qu'il va mourir, se trouve toujours au bord de la route de Lagarce, via de nombreuses références à ses œuvres et à sa personne dans le Journal.

Ai relu Mes parents. Et puis la nuit j'ai écrit à Hervé Guibert une lettre assez longue pour lui dire combien ce livre m'avait bouleversé ${ }^{10}$.

Le projet du "Combat avec l'Enfant, un enfant, tous les enfants, tous les autres... »" vient tout de suite après, avec un titre guibertien : «Ai tenté c'est le mot - une percée sur Le combat avec l'enfant. Ça fait peur ${ }^{12}$. " Le second titre du récit le sera tout autant :

Ai attaqué assez sérieusement (cela me fit un peu peur) ce texte, Le Combat avec

l'enfant. Cela s'appellera Mes deux dernières années (sic !) ${ }^{13}$

Nous devons comprendre : « les deux dernières années de ma vie ». Le dramaturge ne sait pas encore officiellement qu'il est malade - mais il le sait, depuis toujours. Il ne meurt pas prématurément: la mort est toujours déjà là, le virus n'est que la rencontre, l'accomplissement d'une existence malheureuse. La vérité chez lui, l'alethia, le dévoilement, le fait de découvrir, de dénuder, désigne à la fois la mort comme chez les moralistes - dire la vérité à la lumière de la mort - et l'enjeu esthétique de la littérature dire vrai, dire vraiment, dire en vérité. Les enjeux, les finalités semblent les mêmes pour le récit et pour la pièce, comme en atteste le laboratoire littéraire mis en scène dans le Journal : le destin, la genèse des deux textes sont liés, la difficulté à les créer extrême, et soulignée à l'envi. Quelques exemples :

Mais surtout terriblement dire la vérité. Cesser de mentir. [...] Et puis aussi, peutêtre, serait ce enfin l'occasion d'écrire, parce que, ne nous racontons pas d'histoires, jusqu'à présent, n'est-ce pas ${ }^{14} . .$.

Tenter, au milieu du désarroi, de retravailler sur Le Combat avec l'enfant. Mener ça à terme, parce que là est la vérité (même si elle n'est pas bonne à dire). [...] Un déjeuner hier avec la famille, le rebondissement Guibert d'aujourd'hui ne sont-ils pas des signes avant-coureurs ${ }^{15}$ ?

Travail sur Mes deux dernières années. Travail sérieux. Dire la vérité, vraiment. Parfois, je m'éloigne, je raconte une histoire, je triche. Revenir à la difficulté. C'est épuisant ${ }^{16}$.

9 Sur la même page est formulé le projet de Juste la fin du monde, le 11 février 1988 : «Je vais m'atteler très vite à une pièce. Une pièce courte qui me trottait dans la tête depuis quelque temps. Cela s'appelle Les Adieux ${ }^{17}$. » Plus tard, le roman Les Adieux est refusé pour la seconde fois par POL. "Voilà. Ne plus écrire qu'ici? Après Les Adieux, Juste la fin du monde et ce Journal vidéo, comme autant de fins ${ }^{18}$. »

Travail assidu et très volontaire car ça ne vient pas mais alors pas du tout sur Quelques éclaircies. [...] Travail très besogneux sur Quelques éclaircies ${ }^{19}$.

Je tente d'une manière volontaire et quasiment désespérée de travailler sur Quelques éclaircies ${ }^{20}$.

Ai un peu avancé sur Quelques éclaircies, un peu mais ce n'est pas ça, pas ça, pas ça du tout. Complètement délétère sans raison ${ }^{21}$.

Les Adieux correspond au cahier XII du Journal ${ }^{22}$ et au début du cahier XIII On trouve de nombreuses agrafes entre le récit et le Journal. Souvent le récit se loge dans les interstices (les « (...) »). Par exemple, Patricia la femme « aux seins lourds » suscite du dégoût dans le récit ${ }^{23}$, alors que selon le Journal, elle a de "jolis seins $»^{24} \ldots$ ! Ce qu'on perçoit de l'autocensure dans le Journal s'oppose au récit beaucoup plus trash et cru, conformément au projet radical de dire la vérité. 
11 Bref, les réponses sont différentes (Journal, pièce, récit) mais le problème reste identique : gérer la fin, la fin et le posthume: quelle «fin du monde » pour l'auteur? Telle est la grande question du récit Les Adieux, avec sa double proposition pour la fin : alternative du tribunal des hommes qui le maltraitent violemment en énonçant un verdict quant à la vie (soit tout ce qui précède ou à peu près) : « Ils m'ont fait mal, très mal. Ils connaissaient ma vie ils m'en jetaient les morceaux ils m'insultaient », ou la douce vue d'Antoine souriant dans l'encadrement de la porte avec sa question : «Tu as besoin de quelque chose? »

Juste la fin du monde procède aussi de cette interrogation, du «comment finir?» Mais l'opération qui crée le corps du texte restreint, évide, troue, oxygène à la différence du terrible «mur qui tient debout» des Adieux et que le lecteur prend dans la figure. Mais l'opération de création de la version théâtrale ajuste par l'obsession de l'exactitude et de la précision, tout comme Antoine l'ajusteur «construit des outils» et tout comme Catherine à Louis, cherchant le « mot exact » pour l'ajustage : « je ne saurais pas non plus expliquer toutes les petites opérations ${ }^{25} \ldots$.. La série concernant la recherche du réglage de la langue importe dans la pièce: «je ne trouve pas les mots ${ }^{26}$; «sans pouvoir ni savoir jamais rien dire $»^{27}$; «le mot exact $»^{28}$; «Ce n'est pas être méchant, en effet, c'est plus juste ${ }^{29} . »$ (C'est Louis qui parle, juste signifie correct sur le plan grammatical); « simple, claire, précise. Elle énonce bien $»^{30} ;$ «Ils t'expliquent mal » $;$ « mal dit ou trop vite $»^{32} ;$ « juste deux ou trois mots $»^{33}$.

Si l'on revient maintenant au titre de la pièce à la lumière de cette recherche de la justesse, on en trouve la trace dans le titre, augmentée d'une signification supplémentaire. Juste prend le sens de l'exactitude et aussi de la restriction (seulement, uniquement). Juste la fin du monde exprime un renvoi autonymique sur « la fin du monde » et désigne davantage l'expression figée «c'est la fin du monde / ce n'est pas la fin du monde" qu'un événement apocalyptique unique qui serait à la fois une fin, un aboutissement et une révélation (même si le sens religieux continue à vibrer de façon ironique). Le jeu autonymique sur l'expression « fin du monde » la pose donc comme la formule juste du texte. La fin du monde est un objet, un thème, le thème. Ce titre désigne la formule désignant le thème comme exacte, juste, en même temps que minimale - car il va bien falloir se demander ce que l'on soustrait pour en arriver juste à cela? Au-delà, elle désigne la pièce elle-même, comme affaire « rhématique » pour employer la terminologie de Genette, «Juste » fonctionne alors comme le propos que l'on tient sur ce thème, ce que l'on en dit. Le système du titre rend donc compte du jeu de la création de la pièce comme dialectique, dialogue décisif des instances auctoriales et énonciatives au travers des solutions qui s'offrent au personnage de Louis, selon trois étapes.

14 Au commencement, il y aurait d'abord l'hypothèse de la fin du monde - hypothèse simple et sereine. Tout disparaît. Réassurance, remède efficace contre la peur, endormissement général et merveilleux de type " conte de fées ».

15 Puis il y aurait une alternative, autre chose que la fin du monde; la négation, la destruction de la fin du monde. Exit la possibilité d'un effacement irénique. Place à la violence totale et à l'agressivité née de la peur, place à la négativité et au déferlement du mal et à l'anéantissement par la toute puissante méchanceté.

Enfin dépassement, dialectique fine, retour à la position première depuis le savoir gagné du passage par la deuxième position, retour à la création, à la beauté, à la poésie, à l'amour et à l'émotion positive, retour au collectif. Fin de l'empêchement. 
17 Le journal raconte " la patience la douleur et le travail du négatif » et la lutte pour la dialectique, bref le travail de synthèse si l'on veut utiliser la terminologie de Hegel.

J'ai un peu avancé [...], et puis je bute à nouveau, je pense qu'il y a là quelque chose d'important, tout près que je n'arrive pas à atteindre. C'est la première fois que je prends les choses avec autant de clairvoyance, ceci dit. Ce n'est pas bien, je recommence, je recommence. Appliqué. (Trop ?) C'est ma dernière pièce aussi, ou encore, si on veut être plus optimiste: après celle-là, si je la termine, les choses seront différentes ${ }^{34}$.

Ce travail d'élaboration fait l'objet de la scène centrale (scène 10) de la première journée de la pièce qu'on pourrait paraphraser ainsi :

- au début ce que l'on croit que le reste du monde pourrait disparaître avec moi \{position $1:$ l'hypothèse simple\} on se répète à soi-même cette solution comme aux enfants qu'on endort ${ }^{35} \ldots$

- puis l'ironie est revenue... les regarder du ciel \{position 2 : l'ironie, facteur diabolique de division, quelque chose de chimique, un vitriol, va permettre une transformation; négation, destruction\} ;

- je deviens haineux / je dis du mal ! / Je vous détruis sans regret avec férocité / Je vomis la haine elle m'apaise et m'épuise / je vous tue les uns après les autres / Je pense du mal / Je ne vous ai jamais aimé c'était des mensonges ;

- enfin, \{début de réorientation; «à quoi bon ? [...] cesser de jouer »; $3^{\mathrm{e}}$ position, lancée par Mais.\} Alors : " Je traverse à nouveau le paysage en sens inverse. »

19 Ainsi se manifeste un retour éclairé à la position 1, mais affinée, clarifiée, purifiée, au terme de l'opération de décapage par l'acide de l'ironie, le personnage d'Antoine prenant en charge le jugement et la condamnation de la position 2 comme complaisante et aporétique. Selon une telle interprétation, Antoine l'ajusteur n'est pas antagoniste mais secourable et adjuvant, car il seconde efficacement la dynamique soustractive du retour vers l'humanité, la sagesse et la création.

20 Il semble que le récit Les Adieux s'engouffre dans l'ensemble de cette scène, où il coïncide grosso modo avec la position 2, soit la tentation de refuser la fin du monde pour juger définitivement les survivants et les condamner.

Pourquoi est-ce que je fais ça?

Si je conduis jusqu'au bout cette affaire, je couperai tellement de choses, je les détruirai. J'en aurai fini. Ce sera comme mourir, disparaître. En être capable ${ }^{36}$.

21 Tout entier engendré à partir de ce choix, cette posture de l'ironie du mort « mauvais et médiocre » qui manipule les vivants par vengeance pure, le récit inédit intitulé Les Adieux est un monologue irrespirable, tant on en retire l'impression d'une perversité absolument destructrice, sans jeu ni échappatoire possible, où tout est vitriolé par l'acide de l'ironie. Rien ne survit dans cette atmosphère raréfiée, ni amitié, ni amour, ni affection, ni tendresse, ni sérénité : les autres, saisis dans les engrenages de l'impossible machine à dire la vérité, sont presque toujours privés de leurs paroles. Par opposition au dialogue de théâtre, très peu de style direct émerge.

Nicole Collet de Théâtre Ouvert m'a dit beaucoup de bien des Adieux, mais qu'elle avait rarement lu quelque chose d'aussi déprimant ${ }^{37}$.

Le four inaugural de De Saxe, roman, l'échec, la curée forment l'emblème de tout le reste du récit : «Ils attendent la mort du taureau, celle du toréador et l'incendie de l'arène ${ }^{38}$.» Cependant le détour par le récit des Adieux est incontournable à qui veut comprendre l'économie et la dynamique de Juste la fin du monde. 
our conclure, Juste dans le titre est le modalisateur, qui marque que la pièce est le résultat d'une opération, d'une expérimentation comme chimique ou alchimique: il indique le différentiel introduit dans la position 1 par le passage à la position 2 et le retour ultime de la phase 3 : la retraversée du paysage, l'écart par rapport au simple « la fin du monde ». Il permet la création en la désignant comme élaboration, comme jeu fécond, comme mouvement fructueux et non pas comme résultat achevé, produit mortifère, dalle de tombeau.

Ce n'est pas être méchant, en effet,

c'est plus juste ${ }^{39}$.

Nous tenons là la preuve que c'était bien « juste » et que le titre de la pièce désignait bien la pièce elle-même (ce n'était pas la fin du monde, c'était juste la fin du monde) : le même jeu permettra d'engendrer, cinq ans après, Le Pays lointain qui redit le passage initiatique, qui récrit un nouveau retour, et rejoue en l'amplifiant la genèse de Juste la fin du monde. La même dynamique initiatique est prise en charge cette fois, non par Louis, mais par le personnage de l'amant-mort-déjà, ce qui la rend encore plus opératoire.

Faut-il aller plus loin que cette solution philosophique élégante à la question de "Comment créer avec la mort, que faire de la mort? ", que cette construction de la vérité comme passage et comme dialectique? Il est certes utile pour le lire de ne jamais oublier le référentiel philosophique de Jean-Luc Lagarce, même (surtout) s'il le convoque peu explicitement. Faudrait-il, en outre, esquisser un modèle herméneutique d'inspiration protestante qui ferait coïncider dans l'épiphanie de la parole justesse et justice (position $1=$ abandon à la transcendance, position $2=$ captation toute puissante de la transcendance, position $3=$ renoncement à la transcendance) ? Nulle nécessité ici, car selon Juste la fin du monde, la littérature - et elle seule - dit la fin et le retour.

\section{NOTES}

1. Nous ne retenons pas ici l'hypothèse interprétative de Xavier Dolan sur le titre, telle que lisible dans la phrase suivante tirée du film: "C'est un repas de famille, c'est pas la fin du monde ».

2. Professeure émérite, Université de Lille III.

3. Le Voyage de Madame Knipper vers la Prusse orientale y était certes entré avant, en 1982, mais pour des représentations sur la petite scène de l'Odéon.

4. Jean-Luc LAGARCE, Journal, 1977-1990, tome I, 3 septembre 1988, p. 391.

5. Ibid., 9 octobre 1988, p. 406.

6. Jean-LuC LAGARCE, Journal, 1977-1990, tome I, Besançon, Les Solitaires intempestifs, 2007, p. 166.

7. Ibid., p. 211.

8. Ibid., p. 216.

9. Ibid., p. 279.

10. Ibid., 18 septembre 1986, p. 234.

11. Ibid., p. 235.

12. Ibid., 12 janvier 1987, p. 245.

13. Ibid., p. 280.

Sken\&agraphie, 5 | 2018 
14. Ibid., p. 235.

15. Ibid., p. 279.

16. Ibid., p. 282.

17. Id. La formulation est à rapprocher de celle employée à propos du Combat avec l'Enfant : «Ce que je voulais dire (et cela me trotte dans la tête depuis de nombreuses semaines, plusieurs mois à vrai dire),... » (Ibid., p. 235).

18. Jean-LuC LAGARCE, Journal, 1990-1995, tome II, Besançon, Les Solitaires intempestifs, 2008, p. 62.

19. Jean-Luc LAGARCE, Journal, 1977-1990, tome I, op. cit., p. 538.

20. Ibid., p. 539.

21. Ibid., p. 541.

22. Ibid., p. 430.

23. Jean-Luc LAGARCE, Les Adieux, dactylographie inédite, p. 92.

24. Jean-Luc LAGARCE, Journal, 1977-1990, tome I, op. cit., p. 285,

25. Jean-Luc LAGARCE, Juste la fin du monde, Besançon, Les Solitaires intempestifs, «Classiques contemporains », 2012, p. 53.

26. Ibid., p. 50.

27. Ibid., p. 51.

28. Ibid., p. 53.

29. Ibid., p. 54.

30. Ibid., p. 55.

31. Ibid., p. 56.

32. Ibid., p. 57.

33. Ibid., p. 58.

34. Jean-LuC LAGARCE, Journal, 1977-1990, tome I, op. cit, p. 543-544.

35. Jean-Luc LAGARCE, Juste la fin du monde, op. cit, p. 65.

36. Jean-LuC LAGARCE, Journal, 1977-1990, tome I, op. cit, p. 282.

37. Ibid., p. 506.

38. Jean-Luc LAGARCE, Les Adieux, dactylographie inédite, p. 11.

39. Jean-LuC LAGARCE, Juste la fin du monde, op. cit, p. 54.

\section{INDEX}

Mots-clés : Jean-Luc Lagarce, Xavier Dolan, Juste la fin du monde, Le Pays lointain, Les Adieux

\section{AUTEURS}

\section{MARYSE ADAM-MAILLET}

Maryse Adam-Maillet est Inspectrice Pédagogique Régional au rectorat de Franche-Comté et a publié des études consacrées à Zola et Camus. En 2007, elle a organisé la publication du volume collectif Lire un classique du XX $x^{e}$ siècle Jean-Luc Lagarce, coédition Scérén / Solitaires intempestifs. Par ailleurs, elle a publié différents articles sur le français comme langue de scolarisation et sur les élèves allophones. Elle prépare une thèse sur ce sujet. 